\title{
Prognostic and Diagnostic Significance of Tumor Budding Associated with $\beta$-Catenin Expression in Submucosal Invasive Colorectal Carcinoma
}

\author{
Ken Umemura, ${ }^{1}$ Sho Takagi, ${ }^{1}$ Takenobu Shimada, ${ }^{2}$ Takayuki Masuda, ${ }^{2}$ \\ Hisashi Shiga, ${ }^{1}$ Shuichiro Takahashi, ${ }^{1}$ Seiichi Takahashi, ${ }^{1}$ Yoshitaka Kinouchi, ${ }^{1}$ \\ Daisuke Shibuya $^{2}$ and Tooru Shimosegawa ${ }^{1}$ \\ ${ }^{1}$ Division of Gastroenterology, Tohoku University Graduate School of Medicine, Sendai, Miyagi, Japan \\ ${ }^{2}$ Miyagi Cancer Society, Sendai, Miyagi, Japan
}

\begin{abstract}
Endoscopic resection has become a major curative treatment for early colorectal carcinoma without lymph node metastasis. However, lymph node metastasis, a poor prognostic factor in colorectal carcinoma, occurs in about $10 \%$ of the patients with submucosal invasive colorectal carcinoma. Therefore, it is important to identify a high-risk factor for lymph node metastasis in submucosal invasive colorectal carcinoma. This study was designed to identify the relationship between tumor budding with $\beta$-catenin expression and lymph node metastasis in submucosal invasive colorectal carcinoma. We investigated the immunohistochemistry of tumor budding in the 142 patients who underwent surgical resection for submucosal invasive colorectal carcinomas between 1984 and 1999 and the expression pattern of $\beta$-catenin in budding tumor cells. Accordingly, all the patients were followed up for at least 10 years or until death. Among the 142 patients, lymph node metastasis was detected in 14 patients (9.9\%). Univariate analysis showed that tumor budding with $\geq 5$ tumor cells or cell clusters with expression of $\beta$-catenin in the nucleus was significantly associated with lymph node metastasis $(P=0.005)$. In contrast, tumor budding detected by hematoxylin and eosin staining was not associated with lymph node metastasis. Multivariate logistic regression analysis showed that tumor budding with $\geq 5$ tumor cells or cell clusters with expression of $\beta$-catenin in the nucleus was a significant risk factor for lymph node metastasis (odds ratio, $7.124 ; 95 \%$ confidence interval, 1.407-36.062). Thus, tumor budding associated with $\beta$-catenin expression is a risk factor for lymph node metastasis in submucosal invasive colorectal carcinoma.
\end{abstract}

Keywords: $\beta$-catenin; lymph node metastasis; mortality; submucosal invasive colorectal carcinoma; tumor budding Tohoku J. Exp. Med., 2013 Jan, 229 (1), 53-59. C 2013 Tohoku University Medical Press

Endoscopic resection (ER) has become a major curative treatment for early colorectal carcinoma without lymph node metastasis (Kudo 1993; Ueno et al. 2004; Hurlstone et al. 2007). Therefore, the number of cases of ER for early colorectal carcinoma has increased. However, reviews of the literature showed that lymph node metastasis occurred in approximately $10 \%$ of the patients with submucosal invasive colorectal carcinoma (Tanaka et al. 1995; Suzuki et al. 2003). Lymph node metastasis is one of the most important prognostic factors in colorectal carcinoma (Eisenberg et al. 1982; Gennari et al. 2000). Patients with lymph node metastasis should undergo additional radical surgery even after ER. Therefore, it is important to identify the high-risk factors for lymph node metastasis in submucosal invasive colorectal carcinoma.

Possible risk factors for lymph node metastasis in sub- mucosal invasive colorectal carcinoma include the depth of submucosal invasion, poorly differentiated histology, lymphatic vessel invasion, expression of vascular endothelial growth factor-A and $-\mathrm{C}$, and expression of $\beta$-catenin (Takayama et al. 1998; Maeda et al. 2003; Kojima et al. 2005; Walgenbach-Bruenagel et al. 2006; Liang et al. 2006; Saad et al. 2006; Kaneko et al. 2007a; Ishii et al. 2009). Several studies have reported that tumor budding is a histological risk factor for lymph node metastasis of colorectal carcinoma (Hori et al. 2005; Park et al. 2005; Masaki et al. 2006; Kaneko et al. 2007b). Tumor budding is defined as an isolated single cancer cell or a cluster containing $<5$ cancer cells at the invasive front (Fig. 1) (Morodomi et al. 1989; Ueno et al. 2004). Tumor budding is identified with hematoxylin and eosin staining (HE staining) (Kazama et al. 2006). Identification of tumor budding with HE staining is

Received January 24, 2012; accepted November 28, 2012. Published online December 14, 2012; doi: 10.1620/tjem.229.53.

Correspondence: Ken Umemura, Division of Gastroenterology, Tohoku University Graduate School of Medicine, 1-1 Seiryo-machi, Aoba-ku, Sendai, Miyagi 980-8574, Japan.

e-mail: umekun@nyc.odn.ne.jp 


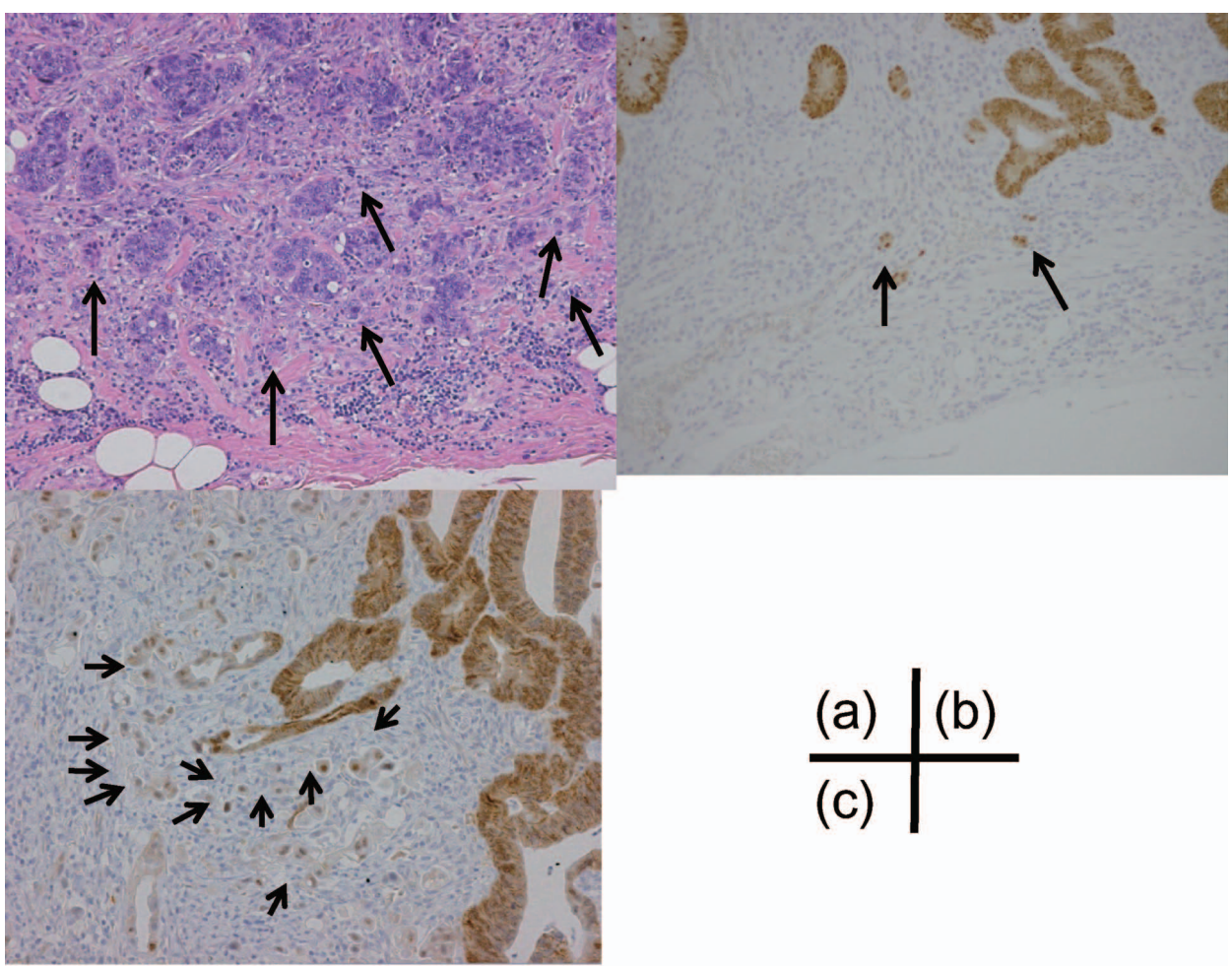

Fig. 1. Representative tumor budding in submucosal invasive colorectal carcinoma. Shown are small clusters of undifferentiated cancer cells or microtubular cancer nests at the invasive margin (original magnification $\times 200$ ). (a) Hematoxylin and eosin staining. Arrows indicate budding cells. (b) $\beta$-catenin staining pattern of the budding cells. Tumor budding with $\geq 1$ tumor cells or cell clusters with positive expression of $\beta$-catenin in the nucleus (Grade 1). (c) $\beta$-catenin staining pattern of the budding cells. Tumor budding with $\geq 5$ tumor cells or cell clusters with positive expression of $\beta$-catenin in the nucleus (Grade 2).

sometimes difficult, and false-positive and false-negative results sometimes occur. The clinicopathological significance of tumor budding detected with anti-cytokeratin antibody is also inconsistent (Jass et al. 2003). There have been several investigations of important molecular events related to tumor budding and $\beta$-catenin activation was shown to contribute to the formation of budding cells at the invasive front (Masaki et al. 2001; Jass et al. 2003). In a previous study, $\beta$-catenin expression was detected in the nucleus and cytoplasm of budding cells (Jass et al. 2003). In the present study, we retrospectively investigated the relationship between tumor budding with $\beta$-catenin expression and tumor budding identified with HE staining. Furthermore, one report investigated the relationship between tumor budding with $\beta$-catenin expression and lymph node metastasis (Masaki et al. 2001). However, no report investigated the relationship between tumor budding with $\beta$-catenin expression in the nucleus and survival prognosis in submucosal invasive colorectal carcinoma. We also investigated the relationship between tumor budding with $\beta$-catenin expression and lymph node metastasis or mortality in patients with submucosal invasive colorectal carcinoma.

\section{Patients and Methods}

Patients

In this study, medical records of patients with colorectal carcinoma were selected from a computerized prospective database at the Miyagi Cancer Society. The prospective database at the Miyagi Cancer Society included the following: (a) clinical characteristics of the patients and their gender and age; (b) relevant pathological features of the tumor, such as size, location, macroscopic appearance, differentiated histology, and lymph node metastasis; (c) the condition at follow-up, operation method, and complications. All patients were followed up by telephone contact once a year. We investigated 142 patients with surgically resected submucosal invasive colorectal carcinomas from the surgical pathology files of the Department of Pathology, Miyagi Cancer Society, Sendai, Japan from April 1984 to March 1999. All patients with submucosal invasive colorectal carcinoma who underwent curative resection at cooperating hospitals of the Miyagi Cancer Society were included retrospectively in this study.

Of these, 64 patients were initially resected by radical surgery, whereas 78 patients were initially resected locally by endoscopic mucosal resection or transanal resection, with additional radical resection being performed. All the patients were followed up for a minimum of one decade, or until death if earlier. We excluded patients with tumors associated with familial adenomatous polyposis or inflammatory bowel disease.

Evaluation of tumor budding in tissue samples

In this study, we investigated tumor budding in tissue samples. 
Tumor budding was evaluated with HE staining and $\beta$-catenin expression. Tumor budding, detected by HE staining, was based on the method reported by Ueno and colleagues (Ueno et al. 2004). Tumor budding was defined as an isolated single cancer cell or a cluster composed of $<5$ cancer cells. After choosing one field where tumor budding was most intensive, the number of budding was counted at the invasive margin in a high power field $(\times 200$ magnification) (Fig. 1). A field with $\geq 5$ buds was considered positive (Ueno et al. 2004). We also evaluated tumor budding associated with $\beta$-catenin expression in tissue samples. The $\beta$-catenin expression pattern is classified according to $\beta$-catenin expression in the nucleus and/or cytoplasm (Jass et al. 2003). One report investigated the relationship between tumor budding with $\beta$-catenin expression in the nucleus and lymph node metastasis (Masaki et al. 2001). However, it was necessary to investigate the relationship between the number of budding cells with positive expression of $\beta$-catenin in the nucleus and lymph node metastasis. Therefore, we categorized tumor budding according to the number of budding cells with positive expression of $\beta$-catenin as follows: Grade 1, tumor budding with $\geq 1$ tumor cell or cell clusters with positive expression of $\beta$-catenin in the nucleus; and Grade 2, tumor budding with $\geq 5$ tumor cells or cell clusters with positive expression of $\beta$-catenin in the nucleus (Fig. 1). Some cases overlapped in each grading of tumor budding. We performed immunohistochemistry with D2-40 to classify lymphatic vessel invasion and tumor budding in the invasive front. D2-40 (mouse monoclonal; DAKO Cytomation, Glostrup, Denmark) was used to identify the lymphatic vessels. Especially, D2-40 is useful for distinguishing the lymphatic vessels from blood vessels (Ishii et al. 2009). The evaluation of tumor budding was performed by two observers who were blind to the clinical data. In case of disagreement, we consulted an independent pathologist. The slides were reviewed, and a consensus view was achieved. Budding positive cases were classified in the budding-positive group, whereas budding negative cases were classified in the budding-negative group.

\section{Evaluation of clinicopathological parameters}

Evaluation of clinicopathological parameters was based on the World Health Organization (Hamilton et al. 2000). Venous invasion was evaluated with HE staining and elastica staining. Lymphatic vessel invasion was evaluated with HE staining and D2-40 staining. We also investigated the depth of submucosal invasion. Using an ocular micrometer, the depth of submucosal invasion was determined as the distance from the bottom of the lamina muscularis mucosae to the invasive front. In cases where the identification of the lamina muscularis mucosae was difficult, the invasive distance was measured as follows: 1, if the macroscopic type was sessile, the distance was measured from the surface of the tumor to the invasive front; and 2, if the macroscopic type was pedunculated with stalk of non-neoplastic mucosa, the distance was measured from a virtual line that linked both ends of the lamina muscularis mucosae to the invasive front (Fig. 2). Details of gender, age, tumor location, tumor size, macroscopic type, and operation method were obtained from the computerized database at the Miyagi Cancer Society.

\section{Immunohistochemistry}

Immunohistochemistry was performed using a standard avidinbiotin-peroxidase technique. We used antibodies to $\beta$-catenin (goat polyclonal; Santa Cruz Biotechnology, Santa Cruz, CA, USA) and D2-40. After deparaffinization and rehydration of 5- $\mu$ m-thick sec- (a)
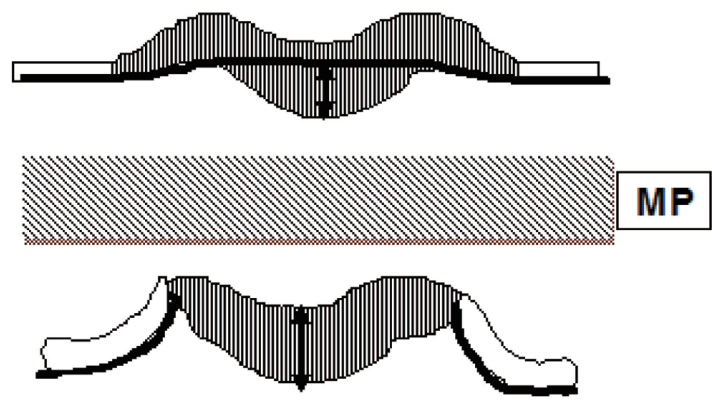

(b)

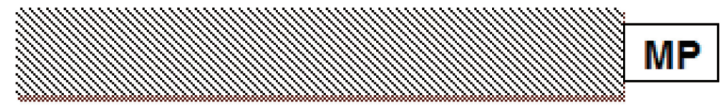

(c)
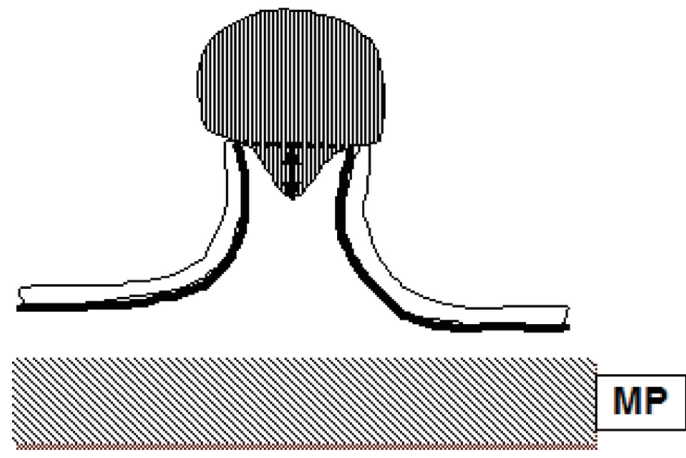

Fig. 2. Methods for measuring the depth of submucosal invasion.

(a) The depth of submucosal invasion was measured from the bottom of the lamina muscularis mucosae to the invasive front. (b) A case of difficult identification. If the macroscopic type was sessile, we measured the distance from the surface of the tumor to the invasive front. (c) A case of difficult identification. If the macroscopic type was pedunculated, we measured the distance from a virtual line that linked both ends of the lamina muscularis mucosae to the invasive front.

tions, the sections were treated with $3 \% \mathrm{H}_{2} \mathrm{O}_{2}$ in $100 \%$ methanol for 30 min to block intrinsic peroxidase activity, and then rinsed in PBS. Sections were autoclaved in $10 \mathrm{mM}$ citrate buffer ( $\mathrm{pH} 9.0$ ) for $40 \mathrm{~min}$ at $90^{\circ} \mathrm{C}$ and allowed to cool at room temperature for more than $1 \mathrm{~h}$. After rinsing the sections in PBS, nonspecific staining was eliminated by incubation with normal goat serum for $10 \mathrm{~min}$. The sections were then incubated with primary antibodies for $1 \mathrm{~h}$, rinsed in PBS, and incubated with secondary antibodies (Nichirei Max-Pro System, Nichirei, Tokyo, Japan) for $30 \mathrm{~min}$. After washing three times with wash buffer, the section products were developed by immersing each in diaminobenzidine tetrahydrochloride solution. Nuclei were lightly counterstained with hematoxylin. The sections were dehydrated and mounted. The positive controls of $\beta$-catenin expression in this study were cancer cells from the invasive front of advanced colorectal carcinoma, and the negative controls were epithelial cells from normal colorectal tissue.

\section{Statistical analysis}

All data were analyzed using SAS version 9.1 (SAS Institute., Inc, Cary, NC, USA) and Stat View-J 5.0 (SAS Institute., Inc, Cary, NC, USA). Fisher's exact test was used to test the correlations between tumor budding and lymph node metastasis. Univariate analysis to compare lymph node metastasis and clinicopathological 
parameters was performed using the chi-square test, Fisher's exact test, and Student's $t$-test. Multivariate analysis with logistic regression was performed to clarify the relative importance of each risk factor for lymph node metastasis. In the latter multivariate analysis, a stepwise method was used to select variables. The results of the multivariate analysis with logistic regression were reported as the odds ratio with $95 \%$ confidence intervals. $P<0.05$ was considered to indicate statistical significance.

In this study, prognosis was calculated overall survival and diseases specific survival. Overall survival and disease specific survival were analyzed using the Kaplan-Meier method. Time to death was calculated from the date of surgery to the date of death or last followup.

The study protocol was approved by the ethics committees of the Miyagi Cancer Society and Tohoku University Graduate School of Medicine, and was conducted in accordance with the declaration of Helsinki.

\section{Results}

Tumor budding in submucosal invasive colorectal carcinoma

Among the 142 patients, 48 were women and 94 were men, and the mean age was 65 (40-79) years. Lymph node metastasis occurred in 14 patients (Table 1). We found tumor budding with $\beta$-catenin expression in 34 patients $(23.9 \%)$. The positive rate of tumor budding associated with $\beta$-catenin expression was as follow: $14.1 \%$ (20 patients at Grade 1) and $9.9 \%$ (14 patients at Grade 2). The positive rate of tumor budding detected with HE staining was $11.3 \%$ (16 patients). Tumor budding with $\beta$-catenin expression was associated with lymph node metastasis. In particular, tumor budding with $\geq 5$ tumor cells or cell clusters with the expression of $\beta$-catenin in the nucleus (Grade 2) was most strongly associated with lymph node metastasis. In contrast, tumor budding detected with HE staining was not associated with lymph node metastasis (Table 1). Of the 14 patients with tumor budding detected with $\beta$-catenin expression (Grade 2), tumor budding was not detectable with HE staining in 7 patients $(50 \%)$. In these seven patients, lymph node metastasis occurred in 2 patients and one patient died.

\section{Univariate analyses}

Univariate analyses of the clinicopathological findings and lymph node metastasis are shown in Table 2. Tumor budding detected by $\beta$-catenin expression (Grade 2) was significantly associated with lymph node metastasis $(P=$ 0.005). In addition, the surgical method was associated with lymph node metastasis $(P=0.048)$. There were no significant correlations between lymph node metastasis and gender, mean age, tumor location, macroscopic type, tumor size, depth of submucosal invasion, blood vessel invasion, lymphatic vessel invasion (HE staining and D2-40 staining), or tumor budding detected by HE staining.

\section{Multivariate analysis}

Multivariate analysis using a logistic regression model showed that tumor budding detected by $\beta$-catenin expression (Grade 2) was significantly associated with lymph node metastasis (odds ratio, 7.124; 95\% confidence interval, 1.407-36.062). In addition, the depth of submucosal invasion was also associated with lymph node metastasis (odds ratio, 1; 95\% confidence interval, 1-1.001). Tumor budding detected by HE staining was not associated with lymph node metastasis (odds ratio, 1.073; 95\% confidence interval, 0.181-6.344) (Table 3).

\section{Decadal mortality in submucosal invasive colorectal carci- noma}

The overall decadal mortality for the 142 patients was 23. Of the 23 cases, 5 were disease-specific death. According to the analysis using Kaplan-Meier method, the overall survival was $83.8 \%$ and the disease-specific survival was $96.5 \%$ in patients with submucosal invasive colorectal carcinoma. In patients with budding-positive lesions detected by $\beta$-catenin expression, the disease-specific decadal survival rate was $100 \%$. In patients with buddingpositive lesions detected by HE staining, there was one disease-specific death. The overall survival curve of patients with submucosal invasive colorectal carcinoma for tumor budding with $\beta$-catenin expression is illustrated in Fig. 3. According to our results, $\beta$-catenin-positive tumor budding did not statistically influence the overall survival.

\section{Discussion}

In this study, we investigated whether immunohistochemical staining of $\beta$-catenin is useful for the detection of tumor budding in submucosal invasive colorectal carcinoma. First, we investigated the incidence of tumor budding in submucosal invasive colorectal carcinoma. One

Table 1. Relation between tumor budding and lymph node metastasis.

\begin{tabular}{cccc}
\hline & \multicolumn{2}{c}{ lymph node metastasis } & $P$ \\
\cline { 2 - 3 } & Positive $(n=14)$ & Negative $(n=128)$ & \\
\hline Budding $(\beta$-catenin) & 5 & 15 & 0.029 \\
Grade 1 & 5 & 9 & 0.005 \\
Grade 2 & 4 & 12 & 0.054 \\
Budding (HE staining) & 4 & \\
\hline
\end{tabular}

(Fisher's exact test) 
Table 2. Relationship between clinicopathological factors and lymph node metastasis.

\begin{tabular}{|c|c|c|c|c|}
\hline & & \multicolumn{2}{|c|}{ lymph node metastasis } & \multirow{2}{*}{$\begin{array}{c}\text { Univariate } \\
P \text { Value }\end{array}$} \\
\hline & & $\begin{array}{l}\text { positive } \\
(n=14)\end{array}$ & $\begin{array}{l}\text { negative } \\
(n=128)\end{array}$ & \\
\hline \multirow[t]{2}{*}{ Gender } & Female & 4 & 44 & 0.773 \\
\hline & Male & 10 & 84 & \\
\hline Mean age ( \pm standard devitation) & & $61.4 \pm 11.7$ & $65.1 \pm 6.9$ & 0.085 \\
\hline \multirow[t]{2}{*}{ Location } & Rectum & 13 & 37 & 0.113 \\
\hline & Colon & 1 & 91 & \\
\hline \multirow[t]{2}{*}{ Macroscopic type } & Pedunculated & 1 & 19 & 0.692 \\
\hline & No pedunculated & 13 & 105 & \\
\hline Size (mean, mm) & & $18.9 \pm 9.7$ & $19.6 \pm 12.7$ & 0.828 \\
\hline \multirow[t]{2}{*}{ Operation method } & Radical resection & 10 & 54 & 0.048 \\
\hline & $\begin{array}{l}\text { Radical resection } \\
\text { after local resection }\end{array}$ & 4 & 74 & \\
\hline Depth of submucosal invasion (mean, $\mu \mathrm{m}$ ) & & $3,628.6 \pm 1,948.7$ & $2,719.5 \pm 2,037.2$ & 0.114 \\
\hline \multirow[t]{2}{*}{ Budding (HE staining) } & positive & 4 & 12 & 0.054 \\
\hline & Negative & 10 & 116 & \\
\hline \multirow[t]{2}{*}{ Budding ( $\beta$-catenin, Grade2) } & positive & 5 & 9 & 0.005 \\
\hline & Negative & 9 & 119 & \\
\hline \multirow[t]{2}{*}{ Blood vessel invasion (HE staining) } & positive & 0 & 3 & $>0.999$ \\
\hline & negative & 14 & 125 & \\
\hline \multirow[t]{2}{*}{ Lymphatic vessel invasion (HE staining) } & positive & 2 & 3 & 0.076 \\
\hline & negative & 12 & 125 & \\
\hline \multirow[t]{2}{*}{ Lymphatic vessel invasion (D2-40) } & positive & 3 & 8 & 0.079 \\
\hline & negative & 11 & 120 & \\
\hline
\end{tabular}

Table 3. Multivariate analysis of risk factors affecting lymph node metastasis.

\begin{tabular}{lccc}
\hline & Odds ratio & $95 \%$ confidence interval & $P$ \\
\hline Gender & 1.154 & $0.291-4.568$ & 0.838 \\
Mean age (years) & 0.918 & $0.841-1.002$ & 0.055 \\
Budding ( $\beta$-catenin, Grade2) & 7.124 & $1.407-36.062$ & 0.018 \\
Budding (HE staining) & 1.073 & $0.181-6.344$ & 0.938 \\
Depth of submucosal invasion (mean, $\mu \mathrm{m})$ & 1 & $1-1.001$ & 0.033 \\
Blood vessel invasion (HE staining) & $<0.001$ & $<0.001->999.999$ & 0.982 \\
Lymphatic vessel invasion (HE) & 0.742 & $0.024-22.701$ & 0.864 \\
Lymphatic vessel invasion (D2-40) & 6.166 & $0.313-121.326$ & 0.232 \\
\hline
\end{tabular}

(Multivariate logistic model)

half of the patients with tumor budding detected with $\beta$-catenin expression were negative by HE staining. Furthermore, in the patients who showed tumor budding with $\beta$-catenin expression but budding negative with $\mathrm{HE}$ staining, lymph node metastasis occurred. In addition, we investigated the relationship between tumor budding with $\beta$-catenin expression and lymph node metastasis in submucosal invasive colorectal carcinoma. We showed that tumor budding with $\beta$-catenin expression in the nucleus of budding cells and many budding cells were significant risk factors for lymph node metastasis in submucosal invasive colorectal carcinoma.
Tumor budding was first described by Morodomi et al. (1989). Tumor budding is defined as an isolated single cancer cell or a cluster composed of $<5$ cancer cells in the invasive front (Morodomi et al. 1989; Ueno et al. 2004). Tumor budding is detected with HE staining and anti-cytokeratin antibody staining. When there are many budding cells at the invasive front, tumor budding can be easily detected with HE staining. However, it is sometimes difficult to detect tumor budding with HE staining, if other cells mask the budding cells. Anti-cytokeratin antibody, expressed by intestinal epithelial cells, can clearly help detect tumor budding even when its detection is difficult 


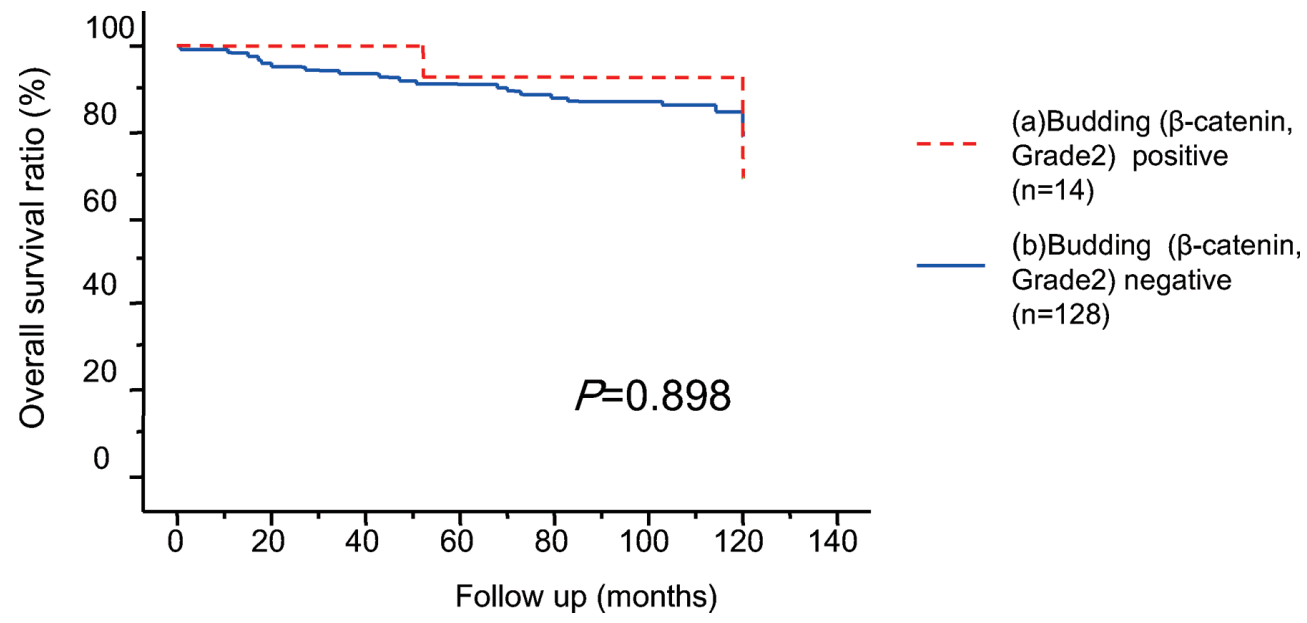

Fig. 3. The overall survival curve of patients with submucosal invasive colorectal carcinoma and tumor budding detected with $\beta$-catenin expression.

The overall survival was not statistically influenced by $\beta$-catenin-positive tumor budding. (a) Budding ( $\beta$-catenin, Grade 2) positive. (b) Budding ( $\beta$-catenin, Grade 2$)$ negative.

with HE staining (Kazama et al. 2006). However, because tumor budding detected with anti-cytokeratin antibody was not associated with lymph node metastasis, its clinicopathological significance remains controversial (Jass et al. 2003). $\beta$-Catenin was first identified as a protein associated with E-cadherin, which maintains cell-cell interactions (Wong et al. 2003). $\beta$-Catenin is a significant factor in the Wnt/ $\beta$-catenin pathway. The $\mathrm{Wnt} / \beta$-catenin pathway is a group of signaling molecules that control a variety of developmental processes, including cell proliferation and cell migration. Altered regulation of Wnt signaling can cause developmental defects and is implicated in the genesis of several human cancers (Miller et al. 1999). When the Wnt/ $\beta$-catenin pathway has an abnormal signal, the epithelial cells accumulate $\beta$-catenin in the nucleus and/or cytoplasm (Morin et al. 1997; Miller et al. 1999; Polakis 2000). The level of $\beta$-catenin expression correlates with the stages of progression in colorectal carcinoma (Wong et al. 2004), and $\beta$-catenin expression at the invasive front is a risk factor for lymph node metastasis in early carcinoma and a poor prognostic factor for disease-specific survival in advanced carcinoma (El-Bahrawy et al. 2001; Utsunomiya et al. 2001; Masaki et al. 2003). These results suggested that $\beta$-catenin is useful for the diagnosis of tumor budding in submucosal invasive colorectal carcinoma. Tumor budding detected with HE staining constituted $50 \%$ of the tumor budding detected with $\beta$-catenin expression, suggesting that cells other than intestinal epithelium might be included in some HE-positive budding cells.

In addition, we investigated the relationship between tumor budding detected by $\beta$-catenin expression and cancerspecific survival in patients with submucosal invasive colorectal carcinoma. According to our data, submucosal invasive colorectal carcinoma patients with tumor budding detected with $\beta$-catenin expression had no disease-specific death after radical surgery. In previous studies, patients with marked tumor budding in stage II-IV colorectal carcinoma had significantly worse survival (Okuyama et al. 2003; Kanazawa et al. 2008). In the present study, no correlation was found between tumor budding and the survival prognosis in submucosal invasive colorectal carcinoma. It will be necessary to perform a prospective study that examines a larger number of local excision or ER cases in submucosal invasive colorectal carcinoma.

In this study, tumor budding with $\beta$-catenin expression was a stronger risk factor for lymph node metastasis. Therefore, we believe that the precise evaluation of tumor budding with $\beta$-catenin will lead to the avoidance of unnecessary surgery in early colorectal carcinoma.

\section{Conflict of Interest}

We declare no conflict of interest during the performance of present study.

\section{References}

Eisenberg, B., Decosse, J.J., Harford, F. \& Michalek, J. (1982) Carcinoma of the colon and rectum: the natural history reviewed in 1704 patients. Cancer, 49, 1131-1134.

El-Bahrawy, M.A., Poulsom, R., Jeffery, R., Talbot, I. \& Alison, M.R. (2001) The expression of E-cadherin and catenins in sporadic colorectal carcinoma. Hum. Pathol., 32, 1216-1224.

Gennari, L., Doci, R. \& Rossetti, C. (2000) Prognostic factors in colorectal cancer. Hepatogastroenterology, 47, 310-314.

Hamilton, S.R. \& Aaltonen, L.A. (2000) World Health Organization classification of tumours: pathology and genetics of tumours of the digestive system. IARC Press, 104-119.

Hori, H., Fujimori, T., Fujii, S., Ichikawa, K., Ohkura, Y., Tomita, S., Ono, Y., Imura, J. \& Kuroda, Y. (2005) Evaluation of tumor cell dissociation as a predictive marker of lymph node metastasis in submucosal invasive colorectal carcinoma. Dis. Colon Rectum, 48, 938-945.

Hurlstone, D.P., Atkinson, R., Sanders, D.S., Thomson, M., Cross, S.S. \& Brown, S. (2007) Achieving R0 resection in the colorectum using endoscopic submucosal dissection. Br. $J$. Surg., 94, 1536-1542.

Ishii, M., Ota, M., Saito, S., Kinugasa, Y., Akamoto, S. \& Ito, I. 
(2009) Lymphatic vessel invasion detected by monoclonal antibody D2-40 as a predictor of lymph node metastasis in T1 colorectal cancer. Int. J. Colorectal. Dis., 24, 1069-1074.

Jass, J.R., Barker, M., Fraser, L., Walsh, M.D., Whitehall, V.L., Gabrielli, B., Young, J. \& Leggett, B.A. (2003) APC mutation and tumour budding in colorectal cancer. J. Clin. Pathol., 56, $69-73$.

Kanazawa, H., Mitomi, H., Nishiyama, Y., Kishimoto, I., Fukui, N., Nakamura, T. \& Watanabe, M. (2008) Tumour budding at invasive margins and outcome in colorectal cancer. Colorectal. Dis., 10, 41-47.

Kaneko, I., Tanaka, S., Oka, S., Kawamura, T., Hiyama, T., Ito, M., Yoshihara, M., Shimamoto, F. \& Chayama, K. (2007a) Lymphatic vessel density at the site of deepest penetration as a predictor of lymph node metastasis in submucosal colorectal cancer. Dis. Colon Rectum, 50, 13-21.

Kaneko, I., Tanaka, S., Oka, S., Yoshida, S., Hiyama, T., Arihiro, K., Shimamoto, F. \& Chayama, K. (2007b) Immunohistochemical molecular markers as predictors of curability of endoscopically resected submucosal colorectal cancer. World $J$. Gastroenterol., 13, 3829-3835.

Kazama, S., Watanabe, T., Ajioka, Y., Kanazawa, T. \& Nagawa, H. (2006) Tumour budding at the deepest invasive margin correlates with lymph node metastasis in submucosal colorectal cancer detected by anticytokeratin antibody CAM5.2. Br. J. Cancer, 94, 293-298.

Kojima, M., Shiokawa, A., Ohike, N., Ohta, Y., Kato, H., Iwaku, K., Hayasi, R. \& Morohoshi, T. (2005) Clinical significance of nuclear morphometry at the invasive front of T1 colorectal cancer and relation to expression of VEGF-A and VEGF-C. Oncology, 68, 230-238.

Kudo, S. (1993) Endoscopic mucosal resection of flat and depressed types of early colorectal cancer. Endoscopy, 25, 455-461.

Liang, P., Hong, J.W., Ubukata, H., Liu, H.R., Watanabe, Y., Katano, M., Motohashi, G., Kasuga, T., Nakada, I. \& Tabuchi, T. (2006) Increased density and diameter of lymphatic microvessels correlate with lymph node metastasis in early stage invasive colorectal carcinoma. Virchows Arch., 448, 570-575.

Maeda, K., Yashiro, M., Nishihara, T., Nishiguchi, Y., Sawai, M., Uchima, K., Onoda, N., Ohira, M., Ishikawa, T. \& Hirakawa, K. (2003) Correlation between vascular endothelial growth factor $\mathrm{C}$ expression and lymph node metastasis in T1 carcinoma of the colon and rectum. Surg. Today, 33, 736-739.

Masaki, T., Goto, A., Sugiyama, M., Matsuoka, H., Abe, N., Sakamoto, A. \& Atomi, Y. (2001) Possible contribution of CD44 variant 6 and nuclear beta-catenin expression to the formation of budding tumor cells in patients with $\mathrm{T} 1$ colorectal carcinoma. Cancer, 92, 2539-2546.

Masaki, T., Matsuoka, H., Sugiyama, M., Abe, N., Sakamoto, A. \& Atomi, Y. (2006) Actual number of tumor budding as a new tool for the individualization of treatment of $\mathrm{T} 1$ colorectal carcinomas. J. Gastroenterol. Hepatol., 21, 1115-1121.

Masaki, T., Sugiyama, M., Matsuoka, H., Abe, N., Izumisato, Y., Goto, A., Sakamoto, A. \& Atomi, Y. (2003) Clinical utility of grading criteria for submucosal invasion in the prognosis of T1 colorectal carcinomas. J. Gastroenterol., 38, 37-44.

Miller, J.R., Hocking, A.M., Brown, J.D. \& Moon, R.T. (1999) Mechanism and function of signal transduction by the Wnt/ $\beta$-catenin and $\mathrm{Wnt} / \mathrm{Ca} 2+$ pathways. Oncogene, 18, 7860-
7872.

Morin, P.J., Sparks, A.B., Korinek, V., Barker, N., Clevers, H., Vogelstein, B. \& Kinzler, K.W. (1997) Activation of $\beta$-catenin-Tcf signaling in colon cancer by mutations in $\beta$-catenin or APC. Science, 275, 1787-1790.

Morodomi, T., Isomoto, H., Shirouzu, K., Kakegawa, K., Irie, K. \& Morimatsu, M. (1989) An index for estimating the probability of lymph node metastasis in rectal cancers. Cancer, 63, 539-543.

Okuyama, T., Oya, M. \& Ishikawa, H. (2003) Budding as a useful prognostic marker in pT3 well- or moderately-differentiated rectal adenocarcinoma. J. Surg. Oncol., 83, 42-47.

Park, S.Y., Choe, G., Lee, H.S., Jung, S.Y., Park, J.G. \& Kim, W.H. (2005) Tumor budding as an indicator of isolated tumor cells in lymph nodes from patients with node-negative colorectal cancer. Dis. Colon Rectum, 48, 292-302.

Polakis, P. (2000) Wnt signaling and cancer. Genes Dev., 14, 1837-1851.

Saad, R.S., Kordunsky, L., Liu, Y.L., Denning, K.L., Kandil, H.A. \& Silverman, J.F. (2006) Lymphatic microvessel density as prognostic marker in colorectal cancer. Mod. Pathol., 19, 1317-1323.

Suzuki, T., Sadahiro, S., Mukoyama, S., Ishikawa, K., Yasuda, S., Tajima, T., Makuuchi, H. \& Murayama, C. (2003) Risk of lymph node and distant metastasis in patients with early invasive colorectal cancer classified as Haggitt's level 4 invasion: image analysis of submucosal layer invasion. Dis. Colon Rectum, 46, 203-208.

Takayama, T., Shiozaki, H., Doki, Y., Oka, H., Inoue, M., Yamamoto, M., Tamura, S., Shibamoto, S., Ito, F. \& Monden, M. (1998) Aberrant expression and phosphorylation of betacatenin in human colorectal cancer. Br. J. Cancer, 77, 605613.

Tanaka, S., Haruma, K., Teixeira, C.R., Tatsuta, S., Ohtsu, N., Hiraga, Y., Yoshihara, M., Sumii, K., Kajiyama, G. \& Shimamoto, F. (1995) Endoscopic treatment of submucosal invasive colorectal carcinoma with special reference to risk factors for lymph node metastasis. J. Gastroenterol., 30, 710-717.

Ueno, H., Mochizuki, H., Hashiguchi, Y., Shimazaki, H., Aida, S., Hase, K., Matsukuma, S., Kanai, T., Kurihara, H., Ozawa, K., Yoshimura, K. \& Bekku, S. (2004) Risk factors for an adverse outcome in early invasive colorectal carcinoma. Gastroenterology, 127, 385-394.

Utsunomiya, T., Doki, Y., Takemoto, H., Shiozaki, H., Yano, M., Sekimoto, M., Tamura, S., Yasuda, T., Fujiwara, Y. \& Monden, M. (2001) Correlation of beta-catenin and cyclin D1 expression in colon cancers. Oncology, 61, 226-233.

Walgenbach-Bruenagel, G., Tolba, R.H., Varnai, A.D., Bollmann, M., Hirner, A. \& Walgenbach, K.J. (2006) Detection of lymphatic invasion in early stage primary colorectal cancer with the monoclonal antibody D2-40. Eur. Surg. Res., 38, 438-444.

Wong, S.C., Lo, E.S., Chan, A.K., Lee, K.C. \& Hsiao, W.L. (2003) Nuclear beta catenin as a potential prognostic and diagnostic marker in patients with colorectal cancer from Hong Kong. Mol. Pathol., 56, 347-352.

Wong, S.C., Lo, E.S., Lee, K.C., Chan, J.K. \& Hsiao, W.L. (2004) Prognostic and diagnostic significance of beta-catenin nuclear immunostaining in colorectal cancer. Clin. Cancer Res., 10, 1401-1408. 